\title{
Outcome of hospitalisation for COVID-19 in patients with interstitial lung disease
}

COVID-19 is a relatively new disease. Adults with certain underlying medical conditions are presumed to be at increased risk for severe COVID-19 illness. Data on the interaction of underlying medical conditions and the risk for severe illness from COVID-19 are currently limited. Identifying these conditions will help to inform clinicians in order to provide the best possible care for patients, and to inform individuals about their level of risk so that they can make decisions about illness prevention. It may also be helpful in identifying those who should be prioritised for vaccination.

Diffuse parenchymal lung diseases (DPLDs), previously referred to as interstitial lung diseases, are a heterogeneous group of disorders of diverse aetiologies and prognosis, while sharing common clinical and pathophysiological features. DPLDs are prone to acute exacerbations with associated increased mortality. Viral infections are thought to be important in triggering exacerbations of DPLDs. COVID-19 is a severe viral pneumonia.

A study by Drake et al. ${ }^{[1]}$ conducted an audit across multiple centres in Europe. Its primary aim was to determine the in-hospital outcome of patients with underlying DPLD hospitalised with COVID-19, comparised with COVID-19 admissions without DPLD, admitted during the same time period and matched for age, gender and comorbidity.

The data analysed comprised 161 admissions with confirmed DPLD and laboratory or clinical evidence of COVID-19 and eligible for propensity score matching with 322 COVID-19 admissions without
DPLD. There was significantly higher mortality in patients with DPLD and COVID-19 compared with those with COVID-19 without DPLD (49\% $(n=79 / 161)$ v. $35 \%(n=114 / 322) ; p=0.013)$. After matching, patients with DPLD with COVID-19 had significantly poorer survival (hazard ratio (HR) 1.60; confidence interval (CI) 1.17 - 2.18; $p=0.003$ ) than age-, sex- and comorbidity-matched controls without DPLD. Patients with a forced vital capacity (FVC) of $<80 \%$ had an increased risk of death compared with patients with FVC $\geq 80 \%$ (HR 1.72; CI 1.05 - 2.83). Furthermore, obese patients with DPLD had an elevated risk of death (HR 2.27; CI 1.39 - 3.71).

The study showed that admitted patients with DPLDs and SARSCoV-2 pulmonary infection were at much higher risk of death, particularly those with an elevated body mass index and poor lung function.

\section{A Chacko}

Division of Pulmonology, Department of Internal Medicine, Sefako Makgatho Health Sciences University and Dr George Mukhari Academic Hospital, Pretoria, South Africa drannachacko@gmail.com

1. Drake TM, Docherty AB, Harrison EM, et al. Outcome of hospitalization for COVID-19 in patients with interstitial lung disease. An international multicentre study. Am J Respir Crit Care Med 2020;202(12):1656-1665. https://doi.org/10.1164/ rccm.202007-2794OC 